\title{
A production recovery plan in manufacturing supply chains for a high-demand item during COVID-19
}

\author{
Sanjoy Kumar Paul
}

UTS Business School, University of Technology Sydney, Sydney, Australia

Email: Sanjoy.Paul@uts.edu.au

Priyabrata Chowdhury

School of Accounting, Information Systems and Supply Chain, RMIT University, Melbourne, Australia

Email: priyabrata.chowdhury@ mit.edu.au

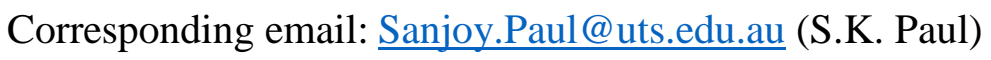

\begin{abstract}
Purpose

A recent global pandemic, known as COVID-19, affects the manufacturing supply chains most significantly. This effect becomes more challenging for the manufacturers of high-demand and most essential items, such as toilet paper and hand sanitizer. In a pandemic situation, the demand of the essential products increases expressively; on the other hand, the supply of the raw materials decreases considerably with a constraint of production capacity. These dual disruptions impact the production process suddenly, and the process can collapse without immediate and necessary actions. To minimize the impacts of these dual disruptions, we aim to develop a recovey model for making a decision on the revised production plan.
\end{abstract}

\section{Design/methodology/approach}

In this paper, we use a mathematical modeling approach to develop a production recovery model for a high-demand and essential item during the COVID-19. We also analyze the properties of the recovery plan, and optimize the recovery plan to maximize the profit in the recovery window.

\section{Findings}

We analyze the results using a numerical example. The result shows that the developed recovery model is capable of revising the production plan in the situations of both demand and supply disruptions, and improves the profit for the manufacturers. We also discuss the managerial implications, including the roles of digital technologies in the recovery process. 


\section{Originality/value}

This model, which is a novel contribution to the literature, will help decision-makers of highdemand and essential items to make an accurate and prompt decision in designing the revised production plan to recover during a pandemic, like COVID-19.

Keywords: Recovery plan; mathematical model; pandemic; COVID-19; supply chains; digital technologies.

\section{Introduction}

Statistics show a strong increasing trend of unexpected and catastrophic events that firms have experienced in the recent past. For example, according to a recent report (Elliott, Thomas and Muhammad, 2019), recording and reporting of such incidents by organizations at its highest level -76.7 percent - in the last years. These events, which are popularly known as disruptions (Tang, 2006; Chen, Sohal and Prajogo, 2013), range from less severe to more severe (Pavlov, Ivanov, Werner, et al., 2019). These disruptions have substantial negative consequences on the return on sales, return on profit, stock return, brand image, employment in the firms, buyers' safety and overall supply chain performance (Hendricks and Singhal, 2003, 2005; Zsidisin, 2003; Thun and Hoenig, 2011; Chowdhury, Lau and Pittayachawan, 2019; Elliott, Thomas and Muhammad, 2019). All these negative consequences are the results of immediate impacts on the supply chain network as one or more components of the network, such as supply, production, distribution or transportation links, become unavailable (Norrman and Jansson, 2004; Shao, 2012; Hishamuddin, Sarker and Essam, 2014; Vergragt, Akenji and Dewick, 2014; Fan and Stevenson, 2018; Ivanov, 2020a). While firms are struggling to manage these firm-or supply chain-specific disruptions, they also have been increasingly experiencing extraordinary outbreaks such as epidemics or pandemics. For example, 1438 epidemics have been reported by the World Health Organization (WHO) between 2010 and 2018 (Hudecheck et al., 2020). The impact of these major outbreaks are more severe for their unique features such as long term existence of the disruptions, ripple effect on other activities, i.e., disruption propagations, high uncertainty and simultaneous impacts on supply, demand, and infrastructure (Choi, 2020; Ivanov, 2020a).

Most recently, since December 2019, firms have been experiencing the major extraordinary outbreak of acute respiratory syndrome coronavirus 2 (SARS-CoV-2), also known as COVID19. Almost all the nations of the world have affected by this outbreak; hence, WHO declared 
this COVID-19 as a pandemic on 11 March 2020 (WHO, 2020). The current impact of this outbreak on the manufacturing firms is already very severe and medium-to-long-term impacts are predicted to be higher than that of any other previous major outbreaks such as 2003 SARS and 2009 H1N1 (Haren and Simchi-Levi, 2020; Koonin, 2020; Laing, 2020; Mogaji, 2020). For example, all the largest 1000 companies in the world have been severely impacted as they all have multiple facilities in the quarantine areas (Linton and Vakil, 2020). Even before declaring this outbreak as a pandemic, production and material supply of 938 of the Fortune 1000 companies have been severely affected given that their tier 1 or tier 2 suppliers are located in Wuhan, China, from where it is generally believed that the COVID-19 originates (Fortune, 2020). Moreover, the severe spread of the virus into Europe and the United States has blocked the movement of the products and materials worldwide (Lee et al., 2020). As such, it has become extremely challenging to continue the operations of supply chains as the operations of some parts of the supply chain has stopped (Breen and Hannibal, 2020; Ivanov, 2020b). While almost all manufacturing firms across various industries have affected by COVID-19 (Linton and Vakil, 2020), the specific nature of the effects varies depending on the nature of the products, e.g., high-demand items or low-demand items. For example, the demand of some products such as toilet paper, hand wash and sanitizers, food items, and medicines goes up expressively while the demand of some other products such as garments and sports items fall drastically (Bagshaw and Powell, 2020; Haren and Simchi-Levi, 2020). During a pandemic, the impacts on the high-demand items are more immediate and visible, given that these products are essential for daily life and, in some cases, for survival (Koyuncu and Erol, 2010; Dasaklis, Pappis and Rachaniotis, 2012; Ivanov, 2020b). Moreover, while firms experience an immediate and sharp increase in demand for these products, they also face a substantial shortage of raw material supply during this pandemic (Ivanov, 2020a; Koonin, 2020; Linton and Vakil, 2020).

Just take an example of a Nowra-based Australian hand sanitizer manufacturing company, Nowchem, which faces both supply and demand disruptions during this COVID-19. While just a few months ago, from December 2019 to February 2020, the company faced a financial hit due to South Coast Bushfire; the company faced unprecedented demand of its product both locally and globally during early March in the face of COVID-19 (Clifford and Huntsdale, 2020). The company ramps up production by utilizing its full capacity and increasing the working and overtime hours of the staffs. However, soon after increasing its capacity, the company started facing shortages of required raw materials such as alcohol, bottles, caps, 
labels, and other ingredients (Alexander, 2020). In fact, not only Nowchem, three-quarters of Australian hand-sanitizer manufacturers reported shortages of key materials such as ethanol, bottle pumps, and sprays. In contrast, more than 50 percent reported shortages of gelling agents, bottles, and pouches (Alexander, 2020). By mid-April of 2020, Nowchem had to stop the production of hand sanitizer due to the shortage of thickening agents. Even, according to the same article (Alexander, 2020), before the complete shutdown of production, the company had been producing 60 percent of its total capacity, 60,000 liters out of 100,000 liters per week. Because of having both supply and demand disruptions, the manufacturing company, Nowchem, should formulate appropriate strategies and recovery plan to recover from this unprecedented event. On the other hand, current literature on disruption recovery strategies and modeling considering an epidemic or pandemic outbreak is mostly limited to humanitarian logistics (Ivanov, 2020a). Therefore, these studies are unable to provide appropriate production recovery model and strategies for commercial products and their supply chains. Considering the inadequacy of research on production recovery modeling considering a major outbreak, this study investigates the following research questions.

i. How can manufacturers make an optimal decision on their production recovery to tackle both supply and demand disruptions caused by a major epidemic or pandemic outbreak like COVID-19?

ii. What are the managerial implications of the developed recovery model?

For answering the above questions, this study develops a mathematical model considering both supply and demand disruptions and optimizes the revised production plan in the recovery window. Using a numerical example, this study demonstrates how the model is capable of optimizing the recovery plan for better tackling the disruption. We simultaneously consider both demand-side disruptions, i.e., sudden demand spikes, and supply-side disruptions, i.e., shortage of material supply, of manufacturing companies. Given that manufacturers of a highdemand item are currently facing both supply shortage and demand boost (Ivanov, 2020a; Lee et al., 2020), it is essential, as well as practical, to consider both the disruptions. In line with the nature of the problem, we also consider the combination of two strategies, increasing production capacity and enhancing raw material supply using emergency sourcing and strong collaboration. Using dual strategies in the presence of dual disruptions makes the model robust and practical (Lücker, Seifert and Biçer, 2019). The main contributions of this study can be summarized as follows. 
i. Developing a mathematical model for production recovery considering the impacts of a pandemic such as COVID-19.

ii. Considering dual disruptions such as increasing demand and shortage of raw material supply in the recovery model.

iii. Analyzing the properties of the model and discussing managerial implications.

The remainder of the paper is organized as follows. Section 2 provides the disruption literature with a focus on major outbreaks and recovery modeling. Section 3 describes the problem and presents the model. The results of the model are analyzed using a numerical example in Section 4 and discussed in Section 5. Managerial implications for the practitioners and contribtions of the study findings are discussed in Section 6. The paper is concluded by summarizing the main insights and outlining the agenda for future research in Section 7.

\section{Literature review}

\subsection{Disruptions and major outbreaks}

It has been reported that the recovery plan for supply chain disruptions varies based on the severity of supply chain disruptions. For example, the recovery plan of less severe and more severe supply chain disruptions is different (Pavlov, Ivanov, Werner, et al., 2019). At the same time, firms need to make a different and more robust plan for an extraordinary supply chain disruption like COVID-19 (Ivanov, 2020a; Ivanov and Dolgui, 2020b). While firms have experience more than 1400 epidemic outbreaks in last ten years, each of which significantly affected operations and productivity of impacted supply chain of commercial products (Blos and Wee, 2020), till to date the research on epidemic outbreaks mostly focused on humanitarian logistics (Ivanov, 2020a). A systematic literature review on the epidemic outbreaks (Dasaklis, Pappis and Rachaniotis, 2012) report that research in this area mostly consider humanitarian logistics to develop several models and strategies to (1) decide the location and number of centers to distribute relief; (2) assign the centers to serve populations within a geographical boundary; (3) select of optimal transportation modes for distributing aid; (4) decide optimal inventory level for commodities and supplies, and (5) formulate replenishment strategies. On the other hand, it is surprising to note that research on commercial products considering epidemic or pandemic outbreaks is scarce.

Even the studies that examine commercial products, mostly investigate the impact of the epidemic outbreaks rather than designing recovery models or strategies for different types of 
products to respond to the outbreak for quick recovery. For example, the economic burden raised from the 2014 Ebola outbreak, which ranges between $\$ 2.8$ and $\$ 32.6$ billion in loss of gross domestic product (GDP) was modeled and reported in Huber, Finelli and Stevens (2018). Similarly, studies indicate the effect of the 2003 SARS outbreak on different contexts such as on the Toronto Pearson International Airport (Johanis, 2007) and the economy of Taiwan, China, and Hongkong (Chou, Kuo and Peng, 2004). In their study Chou, Kuo and Peng (2004) predict a loss of GDP of Taiwan, China, and Hongkong for 0.67, 0.20, and 1.56 percent, respectively. Using a simulation study, a recent article (Ivanov, 2020a) also predicts the impacts of COVID-19, the findings of which conclude that the closing and opening of the facilities at various nodes might become one of the most influential factors that decide the impact on the operations. Another study also suggests that the short-term impacts of COVID19 are already more than all previous outbreaks, including SARS and Ebola, the medium to long-term impact is also predicted as very severe. Still, it is uncertain how severe it would be (Laing, 2020). Recently, Choi (2020) builds an analytical model, explicitly focusing on the distribution side of the supply chain, to show how logistics and technologies together can ensure ' bring-service-near-home' mobile operations from the 'static service operations'.

\subsection{Disruptions and recovery modeling}

In the recent decade, many studies have been carried out on building production recovery models by considering several recovery strategies for managing disruptions. Two main reasons are repetitively mentioned why researchers predominantly focused on formulating recovery models and strategies, rather than mitigating the probability of occurrence, for disruption management. First, disruptions refer to the catastrophic events that are generally hard to predict and control; hence, impossible to eliminate from the operations (Chen, Liu and Yang, 2015; Ivanov et al., 2017). Appropriate recovery strategies are more suitable for tackling these unknown risk events and for making the supply chain resilient (Peck, 2005) and viable (Ivanov, 2020b). Second, firms that failed to implement appropriate recovery strategies failed soon after the disruptions (Cerullo and Cerullo, 2004; Peck, 2005; Chen, Liu and Yang, 2015; Bao, Diabat and Zheng, 2019). For example, 80 percent of the companies experienced by disruptions failed within two years due to poor disruption recovery strategies (Cerullo and Cerullo, 2004).

In building production recovery models, disruptions in all major parts of supply chains, such as the upstream supply side, internal production side, and downstream distribution and demand management side, have been considered. Among them, supply disruptions, such as disruptions 
in supplier facility centers due to fire and machine or system failures and in the sourcing city/country due to natural disasters, political and financial instability, have received the most attention (Shao, 2012; Shao and Dong, 2012; Pal, Sana and Chaudhuri, 2014; Silbermayr and Minner, 2014; Paul, Sarker and Essam, 2016; Darom et al., 2018; Paul and Shams, 2018; Safaeian et al., 2019). Disruptions in the own production facilities, such as machine breakdown, technology obsolescence or breakdown, fire, utility failure, and system damage, have also received considerable focus in building production recovery models (Hishamuddin, Sarker and Essam, 2012; S.K. Paul, Sarker and Essam, 2014; Paul, Sarker and Essam, 2015b, 2015a; Ivanov, 2019). Studies also considered demand side disruptions, such as sudden fall of customers' demand, immediate product obsolesce, in designing the recovery strategies (Asian and Nie, 2014; Sanjoy Kumar Paul, Sarker and Essam, 2014; Ali and Nakade, 2017). In addition to these three major types of disruptions in a supply chain, studies have also developed production recovery model for managing transportation and scheduling disruptions (Hishamuddin, Sarker and Essam, 2013; Fathollahi-Fard, Ranjbar-Bourani, et al., 2019; Paul, Asian, et al., 2019). Some researchers have also considered more than one disruptions as multiple disruptions may happen simultaneously (Ali and Nakade, 2017), or one disruption may affect numerous operational functions due to the ripple effect of supply chain disruptions (Ivanov, Sokolov and Pavlov, 2013; Ivanov, Pavlov and Sokolov, 2014). For example, studies build production recovery model in the presence of dual disruptions, such as supply and transportation disruptions (Hishamuddin, Sarker and Essam, 2015b), supply and demand disruptions (Ivanov, Pavlov and Sokolov, 2014; Sawik, 2019) and three disruptions, such as supply, demand and production disruptions (Paul, Sarker, et al., 2019).

In these studies, researchers proposed several disruption recovery strategies. Among them, backorder (Hishamuddin, Sarker and Essam, 2013, 2015a, 2015b), buffer inventory or safety stock (Darom et al., 2018; Paul and Shams, 2018; Lücker, Seifert and Biçer, 2019), alternative sourcing and backup suppliers (Hou, Zeng and Zhao, 2010; Paul, Sarker and Essam, 2017; Pavlov, Ivanov, Pavlov, et al., 2019), leveraging collaboration and relationship with supply chain partners (Chowdhury, Lau and Pittayachawan, 2016; DuHadway, Carnovale and Hazen, 2017) appropriate compensation policy (Shao and Dong, 2012), spare/reserve capacity (Hishamuddin, Sarker and Essam, 2013; S.K. Paul, Sarker and Essam, 2014), capacity increase or expansion (Ivanov et al., 2016), flexibility (Glenn Richey Jr, Skipper and Hanna, 2009), restructuring of the supply chain such as production-distribution replanning and redesign (Ivanov, Sokolov and Pavlov, 2013; Ivanov, Pavlov and Sokolov, 2014; Ivanov et al., 2016) 
are mostly used. Some studies also proposed a combination of more than one of these strategies (Shao and Dong, 2012; Hishamuddin, Sarker and Essam, 2013; Ivanov et al., 2016; Lücker, Seifert and Biçer, 2019). Depending on the particular scenario and condition, one strategy may be preferred than others. For example, while backup sourcing is a preferred strategy at the beginning of a supply disruption, appropriate policy for compensating customers is more effective as time elapses (Shao and Dong, 2012). Therefore, careful selection of appropriate strategies by considering various factors such as severity, duration and area affected is essential.

\subsection{Knowledge gap}

While existing studies made substantial contributions in the literature in managing less severe to more severe disruptions specific to a particular manufacturing company or supply chain (Pavlov, Ivanov, Werner, et al., 2019), none of these studies considered extraordinary outbreaks such as epidemic or pandemic disruptions, as explained in the previous section. As a result, these production recovery models are not readily applicable to manage a pandemic disruption like COVID-19. Moreover, some of the recovery strategies considered in these studies are not applicable in a pandemic situation for high-demand items. For example, these studies mostly used backorders to develop recovery models. However, backorders are not useful for recovering from a pandemic for high-demand essential items given that quick responding to this demand is required for survival (Dasaklis, Pappis and Rachaniotis, 2012). Besides, during a pandemic situation, firms simultaneously need to enhance the supply of raw materials and production capacity to respond to the increased demand quickly. A combination of these two strategies was not considered in any of the previous mathematical modelings. By considering both strategies, this study demonstrates how a mixture of strategies can be used during a pandemic situation to formulate a production recovery plan for high-demand items that face both demand and supply disruptions.

\section{Problem description and model formulation}

In this paper, we consider a batch production system, which produces a single product with lot size $Q$. In the ideal manufacturing plan, we assume that the annual production rate $(P)$ is higher than the annual demand $(D)$. During the COVID-19, the demand of the product increases substantially due to the necessity of specific products such as toilet paper and hand sanitizer, 
and the raw material supply of such products also reduces considerably as many suppliers unable to supply. Due to having these dual disruptions, it is crucial to design the proper recovery strategies and optimize the production plan accordingly. To develop the recovery model, we consider the following strategies to minimize the impact of the dual disruptions.

Increase in production capacity: During a pandemic, manufacturers of high-demand items can take several actions to increase the production capacity; hence to mitigate the increased demand of a product (Iswara, 2020). For example, a manufacturer can increase the number of shifts, buy additional machinery, utilize the idle time, and hire human resources to increase the production capacity. There is an additional cost for increasing production capacity. We consider the cost for increasing capacity in the recovery model.

Emergency souring and collaboration: During a pandemic, there is a significant shortage of raw material supply from current suppliers, the manufacturer can do emergency sourcing (Huang, Zeng and $\mathrm{Xu}, 2018$ ) from alternative, backup or new suppliers, and leverage collaboration with upstream supply chain partners (Lavastre, Gunasekaran and Spalanzani, 2012) to increase the raw material supply. In this strategy, the raw material price would be higher than the normal situation. We consider this emergency sourcing cost in the recovery model.

In the recovery model, the main objective is to meet the increased demand and to maximize the total profit in the recovery window. We consider a lost sales cost in the objective function for any unmet demand, which will ensure meeting most of the increased demand. To fulfill the objective, we develop a constrained mathematical model, which determines the optimal production plan in the recovery window with maximization of the total profit.

In the following sub-sections, we discuss the mathematical models for the ideal production system and the recovery plan.

\subsection{Model for the ideal production system}

We consider following notations for the ideal production system.

$D \quad$ Annual demand in the ideal plan

$P \quad$ Annual production rate the ideal plan $(P>D)$

$A \quad$ Set-up cost

$H \quad$ Holding cost per unit per year 
$Q \quad$ Lot size in the ideal plan

$T_{c} \quad$ Cycle time $=\frac{Q}{D}$

$T_{S} \quad$ Set-up time

$T_{d} \quad$ Idle time $=\frac{Q}{D}-\frac{Q}{P}-T_{S}$

$R \quad$ Raw material required per cycle $=Q$

We consider an economic production quantity (EPQ) model for the ideal plan. We determine the total annual cost and the optimal lot size of production as follows.

Annual holding cost $=\frac{Q}{2} H \frac{D}{P}$

Annual set-up cost $=\frac{D}{Q} A$

Total annual cost $=\frac{Q}{2} H \frac{D}{P}+\frac{D}{Q} A$

To minimize the total annual cost, $\frac{d}{d Q}\left(\frac{Q}{2} H \frac{D}{P}+\frac{D}{Q} A\right)=0$

After simplifying, the lot size in the ideal production system is calculated as shown in equation (1).

$Q=\sqrt{\frac{2 A P}{H}}$

\subsection{Model for the recovery plan}

We consider following additional notations for the recovery model.

$N \quad$ Number of cycles in the recovery window

$n_{i} \quad$ Times of demand increase in cycle $i$ in the recovery window

$m_{i} \quad$ Times of capacity increase in cycle $i$ in the recovery window

$d_{i} \quad$ Demand in cycle $i$ in the recovery window $=n_{i} \times D \times \frac{Q}{D}=n_{i} Q$

$p_{i} \quad$ Capacity in cycle $i$ in the recovery window $=m_{i} P \times\left(\frac{Q}{P}+T_{d}\right)$ 
$a_{i} \quad$ The fraction of raw material sourced using emergency souring and collaboration in cycle $i$

$b_{i} \quad$ The fraction of raw material sourced from current suppliers in cycle $i$

$r_{i} \quad$ Available raw material in cycle $i$ in the recovery window $=a_{i} R+b_{i} R$

$C_{p} \quad$ Production cost per unit

$C_{c} \quad$ Capacity increase cost

$C_{e} \quad$ Emergency souring cost per unit of raw material

$C_{s} \quad$ Current souring cost per unit of raw material

$L \quad$ Lost sales cost per unit

$S_{p} \quad$ Selling price per unit

$X_{i} \quad$ Production quantity in cycle $i$ in the recovery window

In the recovery plan, we have added several COVID-19 related parameters such as the average cost of increasing production capacity, which includes costs for extra shifts, overtime, hiring staffs, and buying machinery. Another parameter, emergency sourcing cost per unit of raw material, is used to determine the cost for sourcing materials from emerging sources. Lost sales cost, another important parameter in the recovery plan, which considers a penalty cost for any unmet demand, hence the recovery plan will try to reduce the unmet demand to maximize the profit in the recovery plan. These COVID-19 related parameters make the recovery model unique.

In the recovery plan, we have developed mathematical equations for different costs, such as cost to capacity increase, souring, lost sales, holding, and set-up within the recovery window. Production cost is determined by multiplying per unit production cost by total production quantity (equation 2) (Hishamuddin et al., 2014). Capacity increase cost is determined by multiplying the amount of capacity increase by capacity increase cost per shift (equation 3). Lost sales cost (equation 4) is determined by multiplying per unit lost sales cost by total unmet demand (Paul et al., 2015b). Holding cost is calculated as multiplying holding cost per unit per year, average inventory quantity, and time required to keep the inventory (equation 6) (Paul et al., 2014). Set-up cost is calculated by multiplying the cost per set-up with the number of setups in the recovery window (equation 7). 
We have also determined the selling price in the recovery window by multiplying per unit selling price with total production quantities in the recovery window (equation 8). Finally, the total profit is determined by subtracting the total cost from the total selling price in the recovery window.

Production cost $=C_{p} \sum_{i=1}^{N} X_{i}$

Capacity increase cost $=C_{c} \sum_{i=1}^{N} m_{i}$

Sourcing cost $=C_{e}\left(\sum_{i=1}^{N} X_{i}-b_{i} R\right)+C_{s} \sum_{i=1}^{N} b_{i} R$

Lost sales cost $=L\left(\sum_{i=1}^{N} d_{i}-\sum_{i=1}^{N} X_{i}\right)$

Holding cost $=\frac{H}{2 P}\left(\sum_{i=1}^{N} X_{i}^{2}\right)$

Set-up cost $=A N$

Selling price $=S_{p} \times \sum_{i=1}^{N} X_{i}$

The objective function is the maximization of the profit in the recovery window. The total profit $(T P)$ is calculated as presented in equation (9).

$$
\begin{gathered}
\operatorname{Max}(T P)=\text { Total selling price }- \text { total cost } \\
\operatorname{Max}(T P)=S_{p} \sum_{i=1}^{N} X_{i}-C_{p} \sum_{i=1}^{N} X_{i}-C_{c} \sum_{i=1}^{N} m_{i}-C_{e}\left(\sum_{i=1}^{N} X_{i}-b_{i} R\right)-C_{s} \sum_{i=1}^{N} b_{i} R \\
-\frac{H}{2 P}\left(\sum_{i=1}^{N} X_{i}^{2}\right)-A N-L\left(\sum_{i=1}^{N} d_{i}-\sum_{i=1}^{N} X_{i}\right)
\end{gathered}
$$

Subject to the constraints presented in equations (10)-(13).

$\sum_{i=1}^{N} X_{i} \leq \sum_{i=1}^{N} d_{i}$

$X_{i} \leq r_{i} ; \forall i$

$X_{i} \leq p_{i} ; \forall i$

$X_{i} \geq 0 ; \forall i$

Equation (10) represents the constraint for demand, such as total production quantities must be less than or equal to the total demand. The production quantity is restricted by the raw material 
supply, which is presented in equation (11). Equation (12) provides the constraint for production capacity, in which production quantity is also restricted by production capacity. Finally, the non-negativity constraint is shown in equation (13).

\subsection{Analyzing properties of the recovery model}

In this section, we have developed some propositions to describe the properties of the mathematical model developed in section 3.2.

Proposition 1: If $X_{i}=X$ (equal batch sizes in the recovery window), the profit will be reduced as this will consider the minimum capability of production in the recovery window. Hence, equal batch sizes in the recovery window should not be imposed in the recovery window.

Proposition 2: If $C_{c}$ or $C_{e}$ is significantly high (which is most unlikely), and if this negatively impacts the profit lower than the no-action situation, then it is suggested not to implement the strategies.

Proposition 3: if $\sum_{i=1}^{N} d_{i}>\sum_{i=1}^{N} \min \left(r_{i}, p_{i}\right)$, then the lost sales cost will be present in the recovery plan. In this situation, the production quantity $\left(X_{i}\right)$ will be limited by raw material availability or increased production capacity, which will ultimately lead to unmet demand. Hence, there will be lost sales in the recovery plan.

Proposition 4: If $\min \left(r_{i}, p_{i}\right) \geq d_{i}$, then $X_{i}=d_{i}$. In this situation, the raw material availability or increased production capacity is more than the increased demand, and the production process will be capable of recovering fully. Therefore, $X_{i}$ will be equal to the increased demand, $d_{i}$.

Proposition 5: If $\sum_{i=1}^{N} \min \left(r_{i}, p_{i}\right)<\sum_{i=1}^{N} d_{i}$, then $X_{i}=\min \left(r_{i}, p_{i}\right)$. In this situation, the total increased demand is more than the capability in the production, which is $\sum_{i=1}^{N} \min \left(r_{i}, p_{i}\right)$, and the production process will not be capable of recovering fully as $X_{i}$ will be limited by $\min \left(r_{i}, p_{i}\right)$. Hence, $X_{i}$ will be equal to $\min \left(r_{i}, p_{i}\right)$.

\subsection{Solution approach}

There are different mathematical models in operation research, ranging from deterministic single-objective models to multi-objective stochastic models (Fathollahi-Fard, Govindan, et al., 2019; Fathollahi-Fard et al., 2020). The selection of a mathematical model depends on the type of research. As the mathematical model of this study belongs to constrained and non-linear programming, we have applied a Generalized Reduced Gradient (GRG) non-linear approach to solving the model. GRG non-linear approach is capable of handling the constrained and non- 
linear programming model (Eiselt and Sandblom, 2019). The steps of the solution approach are as follows.

Step 1: Input parameters for the ideal plan.

Step 2: Determine the lot size in the ideal plan using equation (1).

Step 3: Input cost and selling price parameters for the recovery plan.

Step 4: Input $n_{i}, m_{i}, a_{i}, b_{i}$ for the recovery plan.

Step 5: Solve the model, presented in Section 3.2, using a GRG non-linear optimization approach.

Step 6: Record the results.

\section{Result analysis}

We analyze the results using a numerical example with hypothetical data for both the ideal production system and the recovery plan. We also compare the results between our model and if the manufacturer does not take any step for recovery, known as no-action situation.

\subsection{Results for the ideal production system}

We assume the following data to determine the ideal plan.

$D=8000$ per year

$P=10000$ per year

$A=\$ 50$ per set-up

$H=\$ 2 /$ unit/year

$T_{S}=0.005$

Lot size, in the ideal plan, is calculated using equation (1) as follows.

$Q=707$ units

We have also calculated cycle time and idle time in a cycle as follows.

Cycle time, $T_{c}=0.088388$

Idle time in a cycle, $T_{d}=0.012678$ 
As we assume, one unit of finished product requires one unit of raw material, and we calculate raw material requirement per cycle as follows.

$R=707$ units

\subsection{Results for the recovery plan}

To analyze the results for the recovery plan, we assume the following hypothetical data.

$\begin{array}{ll}C_{p} & \$ 3 \text { per unit } \\ C_{c} & \$ 2000 \\ C_{e} & \$ 0.5 \text { per unit } \\ C_{S} & \$ 0.2 \text { per unit } \\ L & \$ 8 \text { per unit } \\ S_{p} & \$ 15 \text { per unit } \\ N & 5\end{array}$

The parameters for increased demand, capacity, and raw materials are presented in Table 1.

Table 1: Hypothetical data for increased demand, capacity, and raw material supply

\begin{tabular}{|c|c|c|c|c|c|}
\hline \multirow{2}{*}{ Parameter } & \multicolumn{5}{|c|}{ Cycle } \\
\cline { 2 - 6 } & 1 & 2 & 3 & 4 & 5 \\
\hline$n_{i}$ & 2 & 3 & 2.5 & 1.5 & 2.5 \\
\hline$m_{i}$ & 1.5 & 2 & 2 & 2.5 & 2 \\
\hline$a_{i}$ & 1 & 1 & 1 & 1.5 & 1 \\
\hline$b_{i}$ & 1 & 0.5 & 0.2 & 0.2 & 0.2 \\
\hline$d_{i}$ & 1,414 & 2,121 & 1,768 & 1,061 & 1,768 \\
\hline$r_{i}$ & 1,414 & 1,061 & 849 & 1,202 & 849 \\
\hline$p_{i}$ & 1,251 & 1,668 & 1,668 & 2,085 & 1,668 \\
\hline
\end{tabular}

After solving the model presented in Section 3.2, using GRG non-linear optimization approach, we determine the revised production plan in the recovery window as follows.

$X_{1}=1,251$ units

$X_{2}=1,061$ units

$X_{3}=849$ units

$X_{4}=1,202$ units

$X_{5}=849$ units 
We also calculated different costs and the selling price as follows.

Total production cost $=\$ 15,631.87$

Capacity increase cost $=\$ 20,000$

Sourcing cost $=\$ 2,159.83$

Lost sales cost $=\$ 23,368.84$

Holding cost $=\$ 2,715.06$

Set-up cost $=\$ 250$

Selling price $=\$ 78,159.35$

The value of the objective function (total profit) is optimized as follows.

Total profit $=\$ 14,033.75$

For this numerical example, we have observed that the unmet demand is 2,921 units, which constitute the lost sales cost of $\$ 23,368.84$.

We have also compared the results if the manufacturer does not take any action (no-action situation)for the recovery. In this case, the production plan would be as follows.

$X_{1}=707$ units

$X_{2}=354$ units

$X_{3}=141$ units

$X_{4}=141$ units

$X_{5}=141$ units

The different costs, selling price and total profit would be as follows.

Production cost

$\$ 4,454.77$

Capacity increase cost

Sourcing cost

Lost sales cost

Holding cost

Set-up cost
0

$\$ 296.98$

$\$ 53,174.43$

$\$ 250$
$\$ 68.5$ 
Selling price

$\$ 22,273.86$

Total profit

$-\$ 35,970.8$ (loss)

In this no-action situation, the lost sales cost is significantly high compared to the recovery model, as the manufacture was not able to meet the demand, which was lost. The total unmet demand is 6,647 units, which constitute the total lost sales cost of $\$ 53,174.43$. If the manufacturer does not take any steps for the recovery plan, the total profit would substantially reduce to $-\$ 35,970.8$, which is a loss. By applying the recovery strategies and our model, the improvement in total profit is significant.

\section{Discussion on findings}

The findings from our developed model and results are discussed in this section.

Impact of recovery strategies: Both of the proposed strategies have a positive effect on the recovery plan. However, they should be implemented together. An increase in production capacity will ensure the increment in production quantity to meet the increasing demand. Emergency sourcing and collaboration will help to increase the supply of raw materials, which is another requisite for producing additional amounts.

Impact of lost sales cost: if any manufacturer fails to meet the demand, there will be a cost for this. The cost of this unmet demand is high if the manufacture does not have any recovery strategy. Our proposed model will help to reduce these lost sales cost significantly by lowering the unmet demand during the recovery process. However, the total profit in the recovery window will decrease with the increment of lost sales cost $(L)$, as shown in Figure 1.

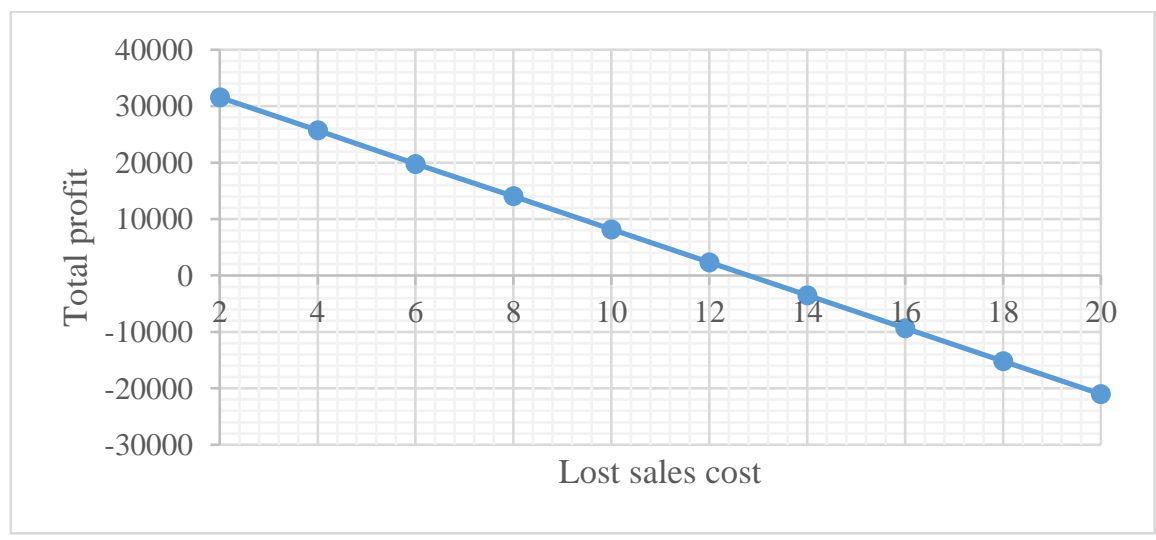

Fig. 1: Impact of lost sales cost on the total profit during recovery

Changes in the total profit: From the results of our proposed model, we can see significant improvement in profit, compared to the no-action taken. The total profit mainly depends on the 
capability of meeting increased demand. However, other costs, such as cost of increasing capacity, cost for emergency sourcing, and lost sales cost, play an essential role in the total profit function. Figures 2 and 3 show the impacts of capacity increase cost and sourcing cost, respectively, on the total profit. Other cost parameters, such as holding cost and set-up, have little impact on the total profit. The impact of holding cost on the total profit in the recovery window is presented in Figure 4. Total profit in the recovery plan decreases with the rise of capacity increase cost, sourcing cost, and holding cost. However, it was observed that if capacity increase cost is more than a certain level (e.g., it was \$5, 000 in our numerical example - see Figure 2), then the total profit reduces below the no-action taken situation. Therefore, manufacturers should be careful with the trade-off of capacity increase cost with the overall profit while making the decision.

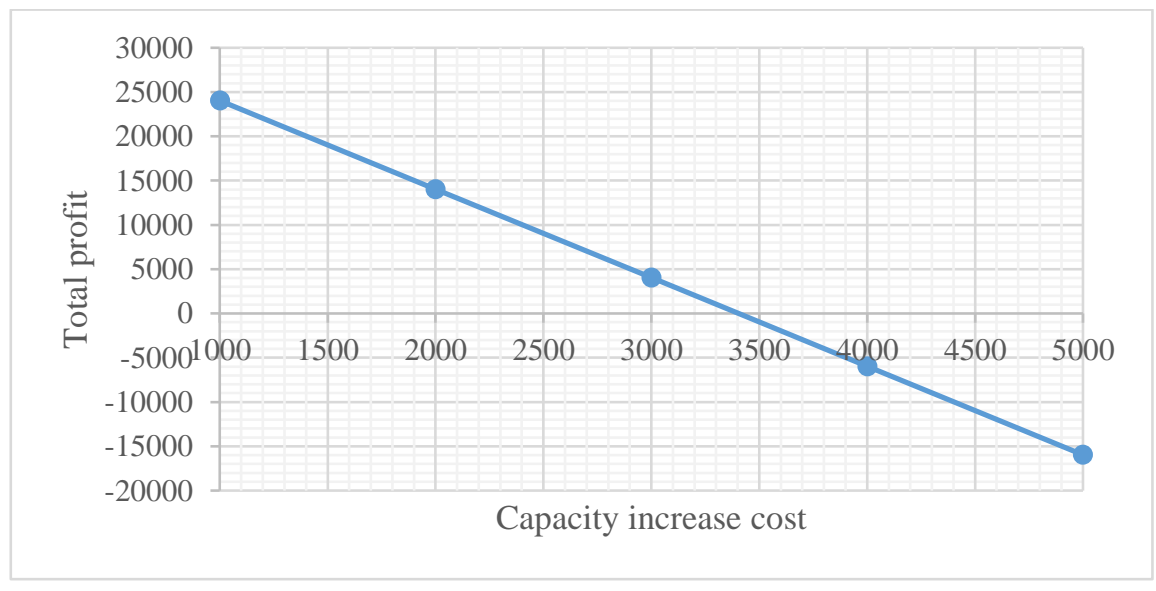

Fig. 2: Impact of capacity increase cost on the total profit during recovery

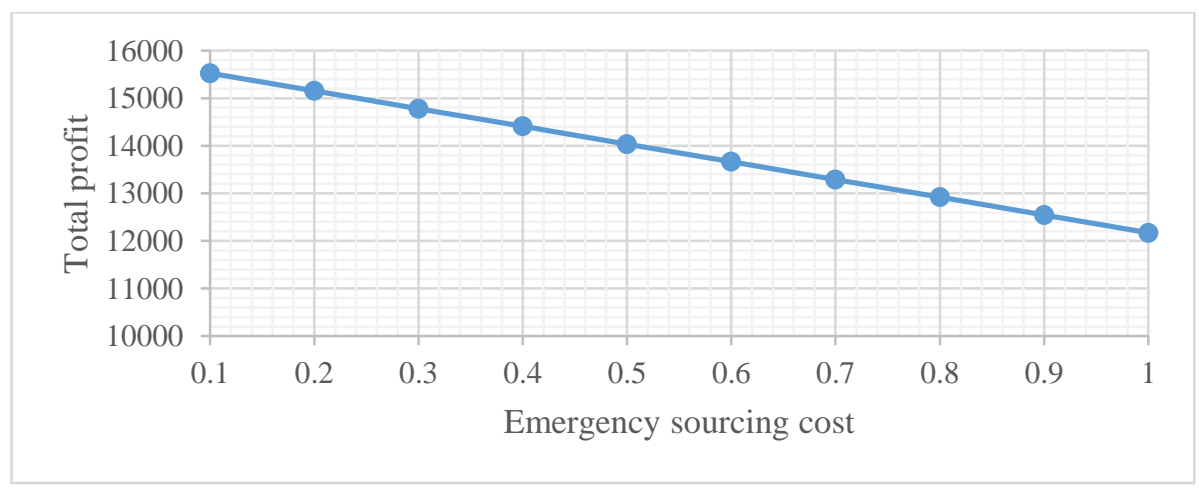

Fig. 3: Impact of emergency sourcing cost on the total profit during recovery 


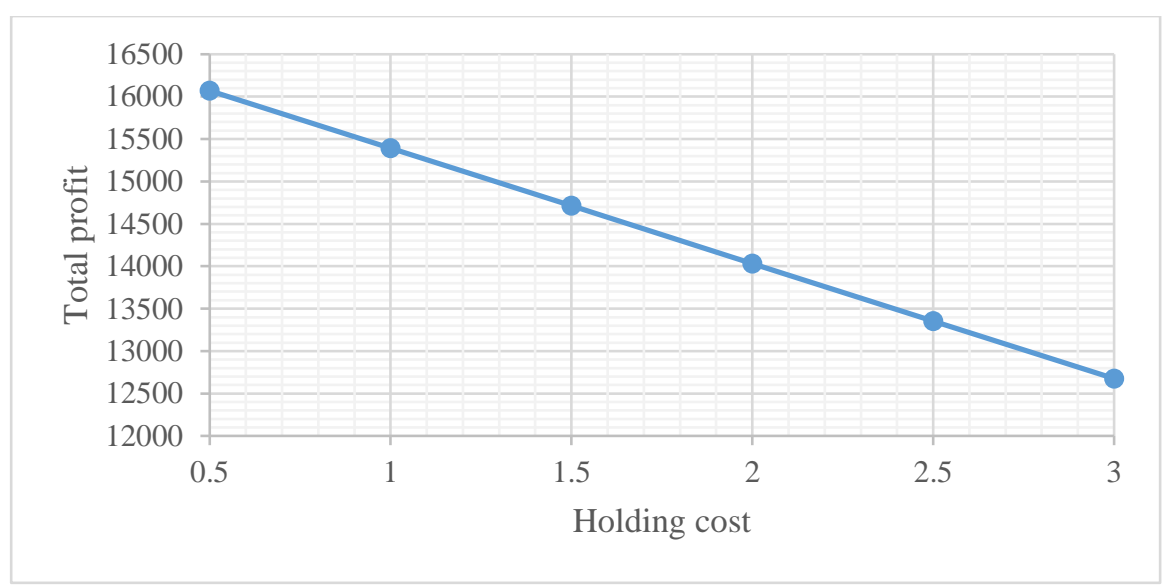

Fig. 4: Impact of holding cost on the total profit during recovery

Impact of $\boldsymbol{n}_{\boldsymbol{i}}, \boldsymbol{m}_{\boldsymbol{i}}, \boldsymbol{a}_{\boldsymbol{i}}$, and $\boldsymbol{b}_{\boldsymbol{i}}$ : Total profit in the recovery window also depends on the changed scenarios such as the value of $n_{i}, m_{i}, a_{i}$, and $b_{i}$. Table 2 shows the comparison of results for different values of $n_{i}, m_{i}, a_{i}$, and $b_{i}$. In this analysis, we have changed the value of one parameter and fixed the values of others. It is observed that our model performs better than the no-action situation in all scenarios.

Table 2: Impact of $n_{i}, m_{i}, a_{i}$, and $b_{i}$

\begin{tabular}{|c|c|c|c|}
\hline \multirow{4}{*}{$\begin{array}{c}\text { Fixed values } \\
m_{i}=2\end{array}$} & $n_{i}$ & Total profit (our model) & Total profit (no-action) \\
\cline { 2 - 4 }$a_{i}=1$ & 1.5 & 38455.25 & -7737.05 \\
\cline { 2 - 4 }$b_{i}=0.5$ & 2 & 24313.25 & -21879 \\
\cline { 2 - 4 } & 2.5 & 10171.25 & -36021 \\
\cline { 2 - 4 } & 3 & -3970.75 & -50163 \\
\cline { 2 - 4 } Fixed values & 3.5 & -18112.70 & -64305 \\
$n_{i}=2$ & 4 & -32254.70 & -78447 \\
$a_{i}=1$ & $m_{i}$ & Total profit (our model) & Total profit (no-action) \\
\cline { 2 - 4 }$b_{i}=0.5$ & 2.5 & 29313.25 & -21879 \\
\cline { 2 - 4 } & 2.5 & 24313.25 & -21879 \\
\cline { 2 - 4 } Fixed values & 3 & 19313.25 & -21879 \\
$n_{i}=2$ & $a_{i}$ & 14313.25 & -21879 \\
$m_{i}=2$ & 0.5 & -8595.40 & -21879 \\
$n_{i}=0.5$ & 1 & 24313.25 & -21879 \\
\cline { 2 - 4 } & 1.5 & 56596.92 & -21879 \\
\cline { 2 - 4 } & 2 & 56596.92 & -42827.4 \\
\hline \multirow{4}{*}{ Fixed values } & $b_{i}$ & Total profit (our model) & Total profit (no-action) \\
\cline { 2 - 4 }$n_{i}=2$ & 0.2 & 4324.86 & -28856.8 \\
\cline { 2 - 4 }$m_{i}=2$ & 0.4 & 17675.46 & -14906.3 \\
\cline { 2 - 4 }$a_{i}=1$ & 0.6 & 30926.05 & -975.68 \\
\cline { 2 - 4 } & 0.8 & 44076.65 & 12934.9 \\
\cline { 2 - 4 } & 1 & 57127.25 & \\
\hline
\end{tabular}




\section{Managerial implications and theoretical contributions}

The model and strategies, developed in this paper, can be applied in determining a recovery plan for a high-demand and essential product, such as toilet paper and hand sanitizer, during a pandemic like COVID-19. The COVID-19 outbreak creates dual disruptions such as a sudden increase in demand substantially and a decrease in raw material supply in supply chains of a high-demand item (Ivanov, 2020b; Koonin, 2020). In addition, there is a limitation of production capacity. We have considered all of these impacts of COVID-19 on the supply chain of a high-demand item in the developed model, which make the model realistic, robust and practical. Managers can use the concept and model to generate a revised production plan. Hence, it can be said that this study contributes to the practice by providing an implementable production recovery model to manage the impacts of a pandemic like COVID-19 on global supply chains. In this research, the model is found effective in improving the total profit in the recovery window. Moreover, proper implementation of the model can help managers of the supply chains of a high-demand item to remain viable during the recovery process, as suggested in Ivanov (2020b).

In this paper, we have considered two recovery strategies (increase in production capacity and increase in raw material supply) to develop the recovery model. Managers can take several actions to implement these strategies, such as an increase in production shifts, use of spare capacity, buying new machinery, and hiring human resources to increase production capacity and emergency sourcing from available suppliers and collaborations with supply chain partners to increase the raw material supply. As the results suggest, practitioners need to improve both strategies simultaneously as those are the constraints in the decision making model. If a manager implements one strategy out of the proposed two, the recovery plan may not be useful (Shao and Dong, 2012). For example, in a situation when managers only increase the raw materials supply without enhancing production capacity, then the additional materials will only create buffer materials and increase the operating cost for the companies (Wilson, 2007). Hence, to get the maximum benefits from the model, decision makers are suggested to implement both strategies and determine the recovery plan using the proposed model as soon as possible they experienced the impact of a pandemic.

As an option to increase production capacity, managers can increase the number of production hours by introducing the second or even third shift each day or simply increasing the current production hours. For example, Kimberly-Clark, the leading toilet paper producer in Australia, 
runs 24 hours at its South Australian factory to respond to the increased demand (Bagshaw and Powell, 2020). As we consider, ideally, the production capacity is greater than the demand rate; hence there is some spare capacity in the system, which managers can utilize to increase the capacity. however, these require additional workforce and extra maintenance of machinery. It is also possible to buy additional machinery to use other production facilities to increase production capacity. Managers should also try using emergency sourcing options to increase the raw material supply. This emergency sourcing includes utilization of supply capacities of current suppliers, alternative and backup suppliers, and sourcing from new suppliers. Moreover, collaboration and information sharing with supply chain partners such as other distributors and suppliers could help to find new sourcing options and ultimately help to increase the raw material supply (Cheong and Song, 2013).

In this model, we have considered lost sales costs, which determines the cost of the unmet demand during the recovery. The cost of this lost sales is an important parameter, and managers should be careful to determine the accurate data for lost sales costs. Lost sales cost per unit should not be greater than the per unit selling price because this is the cost of not meeting demand, which cannot be greater than the selling price. Finally, to recover for a high-demand item during a pandemic, it is vital to implement the recovery strategies quickly (Chen, Liu and Yang, 2015; Ivanov, 2020a), although, in real-life cases, it is a challenging task to implement them in a speedy manner. As such, we urge managers to be proactive in looking for actions for implementing the two strategies proposed in this study.

Moreover, managers can take advantage of digital technologies as these technologies can play a significant role in implementing the strategies and recovery plan suggested in this study. For example, this study suggests better supply chain collaboration, which requires on-time sharing of accurate information between buyers and suppliers (Chowdhury, Lau and Pittayachawan, 2019), to improve raw material supply. In this regard, data analytics and blockchain can be employed to improve the supply chain visibility (Ivanov and Dolgui, 2020a), thereby, enhancing collaborations with supply chain partners. Moreover, blockchain systems can assist in keeping the data needed for recoveries such as information and data for production capacity, human resources requirements, and information of supplier capacities, and emergency suppliers. Hence, managers can use the data and information to undertake actions for implementing the developed recovery model. Another digital technology, additive manufacturing, can also help to implement the proposed recovery strategies quickly by utilizing its reserved inventory and capacity and by identifying and maintaining contingent suppliers for 
emergency sourcing (Ivanov, Dolgui and Sokolov, 2019). A recent empirical study (Das, Gottlieb and Ivanov, 2019) has also supported the application of digital technologies, such as Industry 4.0, blockchain, IoT and additive manufacturing, for improving the recovery capabilities in the recovery window. Therebefore, we suggest that managers use digital technologies to ensure the successful implementation of the recommended strategies.

The main contribution of this study lies in the development of a production recovery model for the high-demand items during a major pandemic outbreak. A recent study (Ivanov, 2020a) focusing on COVID-19 clearly states that the current body of literature using major epidemic or pandemic outbreaks mainly focused on humanitarian logistics but ignored commercial companies. On the other hand, the current COVID-19 pandemic outbreak is a unique disruption and an extraordinary situation, which is different from any other previous disruptions (Ivanov and Dolgui, 2020b). As such, developing a production recovery model focusing on this extraordinary outbreak for the high-demand commercial products can enhance the current knowledge. Moreover, the study demonstrates how mathematical modeling can be used to develop a recovery plan by accommodating several actions such as increasing the number of production shifts, buying or hiring machinery, hiring human resources, emergency sourcing and collaboration with supply chain partners. These actions ultimately help to achieve two broad strategies, i.e., an increase in production capacity and an increase in raw material supply, in the presence of both demand-side and supply-side disruptions during a pandemic. Such a robust model is a unique contribution of this study as using mathematical modeling; none of the previous research on disruption management has considered these two strategies and two large-scale disruptions simultaneously. In addition, the outcome of this study, a robust and practical recovery model, can substantially assist practitioners of commercial high-demand products in designing a production recovery plan for a quick recovery during a pandemic situation.

\section{Conclusions}

COVID-19 is an exceptional and extraordinary event that impacts the supply chain globally. The challenge for the manufacturers of high-demand and the essential product has twofold: i) the demand of the product increases substantially and suddenly, ii) the supply of the raw material decreases without notice. These dual disruptions make the production planning complex, and without proper action, the business could be unable to ramp up the production and could lose the demand. This research tackles both of these disruptions and develops a 
recovery model to revise the production plan, for a certain time in the future - known as the recovery window, to maximize the total profit. In this mathematical recovery model, we consider an increase in production capacity and emergency sourcing and collaboration as recovery strategies. Our research finds that there are significant improvements in the total profit if manufacturers can implement both recovery strategies simultaneously. This research supplements the inadequate studies on developing mathematical models and strategies for production recovery, considering the impact of an epidemic or pandemic situation.

As CVOID-19 is a new experience for supply chain decision makers, they would face numerous challenges to decide on recovery planning. The model, developed in this paper, could be a base paper for decision makers to make a recovery decision. Moreover, this paper provides a mathematical model and numerical results, which could be useful to understand the impact of the COVID-19 and formulate recovery strategies.

The developed recovery model, in this research, is only applicable for revising the production plan. While this study substantially contributes to the literature on the production recovery plan for high-demand commercial products during a major outbreak such as an epidemic or a pandemic, in the future, the concept can be further extended to develop a recovery plan in a complex and global supply chain network considering the impact of a global pandemic like COVID-19. This extension will help to formulate the strategies to revise the supply, manufacturing, and distribution plans simultaneously in the supply chain. In this paper, we use hypothetical data to analyze the recovery plan. future studies may consider collecting real data from specific supply chains, such as supply chains of food and medicine products, to develop and analyze the recovery model. Such an extension could potentially allow the researchers to consider product-specific parameters in the model formulation. Furthermore, a future study could investigate the recovery models for low-demand items such as garments or athletic products during a pandemic as the current model only considers the high-demand items. Besides, full empirical studies, such as in-depth case studies or a large-scale survey, can be conducted to provide an in-depth understating of how the proposed strategies help recover or to validate the proposed strategies and their impact on the profit.

\section{References}

Ali, S. M. and Nakade, K. (2017) 'Optimal ordering policies in a multi-sourcing supply chain with supply and demand disruptions-a CVaR approach', International Journal of Logistics Systems and Management, 28(2), pp. 180-199. 
Asian, S. and Nie, X. (2014) 'Coordination in Supply Chains With Uncertain Demand and Disruption Risks : Existence, Analysis , and Insights', IEEE Transactions on Systems, Man, and Cybernetics: Systems, 44(9), pp. 1139-1154.

Bagshaw, E. and Powell, D. (2020) 'Supermarkets stockpile, toilet paper production runs 24 hours', The Sydney Morning Herald, 3 March.

Bao, X., Diabat, A. and Zheng, Z. (2019) 'An ambiguous Manager's disruption decisions with insufficient data in recovery phase', International Journal of Production Economics. Elsevier B.V., Article in. doi: 10.1016/j.ijpe.2019.07.038.

Blos, M. F. and Wee, H.-M. (2020) 'A supply chain vulnerability map for the Automotive and Electronic industries in Brazil', International Journal of Service Management and Sustainability, 3(2), pp. 83-95. doi: 10.24191/ijsms.v3i2.8107.

Breen, L. and Hannibal, C. (2020) 'Learning from the Covid-19 pandemic : planning, controlling and driving change for greater resilience in supply chains: Special issue call for papers', Supply Chain Management: An International Journal.

Cerullo, V. and Cerullo, M. J. (2004) 'Business continuity planning: a comprehensive approach', Information Systems Management, 21(3), pp. 70-78. doi: 10.1201/1078/44432.21.3.20040601/82480.11.

Chen, J., Sohal, A. S. and Prajogo, D. I. (2013) 'Supply chain operational risk mitigation: a collaborative approach.', International Journal of Production Research, 51(7), pp. 21862199. doi: 10.1080/00207543.2012.727490.

Chen, L.-M., Liu, Y. E. and Yang, S.-J. S. (2015) 'Robust supply chain strategies for recovering from unanticipated disasters', Transportation Research Part E: Logistics and Transportation Review. Elsevier Ltd, 77, pp. 198-214.

Cheong, T. and Song, S. H. (2013) 'The value of information on supply risk under random yields', Transportation Research Part E: Logistics and Transportation Review. Elsevier Ltd, 60, pp. 27-38. doi: 10.1016/j.tre.2013.09.006.

Choi, T. (2020) 'Innovative "Bring-Service-Near-Your-Home" operations under CoronaVirus ( COVID-19 / SARS-CoV-2 ) outbreak : can logistics become the messiah?', Transportation Research Part E. Elsevier Ltd. doi: 10.1016/j.tre.2020.101961.

Chou, J., Kuo, N.-F. and Peng, S.-L. (2004) 'Potential impacts of the SARS outbreak on 
Taiwan's economy', Asian Economic Papers, 3(1), pp. 84-99. doi: $10.1162 / 1535351041747969$.

Chowdhury, P., Lau, K. H. and Pittayachawan, S. (2016) 'Supply risk mitigation of small and medium enterprises: a social capital approach', in The Proceedings of 21st International Symposium on Logistics. Centre for Concurrent Enterprise, Nottingham University, pp. 37 44.

Chowdhury, P., Lau, K. H. and Pittayachawan, S. (2019) 'Operational supply risk mitigation of SME and its impact on operational performance: a social capital perspective', International Journal of Operations \& Production Management, 39(4), pp. 478-502.

Darom, N. A. et al. (2018) 'An inventory model of supply chain disruption recovery with safety stock and carbon emission consideration', Journal of Cleaner Production, 197, pp. 1011-1021. doi: 10.1016/j.jclepro.2018.06.246.

Das, A., Gottlieb, S. and Ivanov, D. (2019) 'Managing disruptions and the ripple effect in digital sup-ply chains: empirical case studies, ', in Ivanov, D., Dolgui, A., and Sokolov, B. (eds) Handbook of the Ripple Effects in the Supply Chain. Springer, New York, pp. 261-285.

Dasaklis, T. K., Pappis, C. P. and Rachaniotis, N. P. (2012) 'Epidemics control and logistics operations: a review', International Journal of Production Economics. Elsevier, 139(2), pp. 393-410. doi: 10.1016/j.ijpe.2012.05.023.

DuHadway, S., Carnovale, S. and Hazen, B. (2017) 'Understanding risk management for intentional supply chain disruptions: risk detection, risk mitigation, and risk recovery', Annals of Operations Research. Springer US, pp. 1-20. doi: 10.1007/s10479-017-2452-0.

Eiselt, H. A. and Sandblom, C.-L. (2019) 'Methods for nonlinearly constrained problems', in Nonlinear Optimization. Springer, pp. 243-278. doi: 10.1007/978-3-030-19462-8_7.

Elliott, R., Thomas, C. and Muhammad, K. (2019) BCI supply chain resilience report. London: Business Continuity Institute.

Fan, Y. and Stevenson, M. (2018) 'A review of supply chain risk management: definition, theory, and research agenda', International Journal of Physical Distribution \& Logistics Management, 48(3), pp. 205-230.

Fathollahi-Fard, A. M., Govindan, K., et al. (2019) 'A green home health care supply chain: new modified simulated annealing algorithms', Journal of Cleaner Production. Elsevier Ltd, 
240, p. 118200. doi: 10.1016/j.jclepro.2019.118200.

Fathollahi-Fard, A. M., Ranjbar-Bourani, M., et al. (2019) 'Novel modifications of social engineering optimizer to solve a truck scheduling problem in a cross-docking system', Computers and Industrial Engineering. Elsevier, 137, p. 106103. doi:

10.1016/j.cie.2019.106103.

Fathollahi-Fard, A. M. et al. (2020) 'An adaptive Lagrangian relaxation-based algorithm for a coordinated water supply and wastewater collection network design problem', Information Sciences. Elsevier Inc., 512, pp. 1335-1359. doi: 10.1016/j.ins.2019.10.062.

Fortune (2020) Fortune 1000. Available at: https://fortune.com/2020/02/21/fortune-1000coronavirus-china-supply-chain-impact/ (Accessed: 20 April 2020).

Glenn Richey Jr, R., Skipper, J. B. and Hanna, J. B. (2009) 'Minimizing supply chain disruption risk through enhanced flexibility', International Journal of Physical Distribution \& Logistics Management, 39(5), pp. 404-427.

Haren, P. and Simchi-Levi, D. (2020) 'How Coronavirus could impact the global supply chain by mid-March', Harvard Business Review.

Hendricks, K. B. and Singhal, V. R. (2003) 'The effect of supply chain glitches on shareholder wealth', Journal of Operations Management, 21(5), pp. 501-522.

Hendricks, K. B. and Singhal, V. R. (2005) 'Association between supply chain glitches and operating performance', Management Science, 51(5), pp. 695-711.

Hishamuddin, H., Sarker, R. A. and Essam, D. (2012) 'A disruption recovery model for a single stage production-inventory system', European Journal of Operational Research. Elsevier B.V., 222(3), pp. 464-473.

Hishamuddin, H., Sarker, R. A. and Essam, D. (2013) 'A recovery model for a two-echelon serial supply chain with consideration of transportation disruption', Computers \& Industrial Engineering, 64(2), pp. 552-561.

Hishamuddin, H., Sarker, R. A. and Essam, D. (2014) 'A recovery mechanism for a two echelon supply chain system under supply disruption', Economic Modelling. Elsevier B.V., 38 , pp. 555-563.

Hishamuddin, H., Sarker, R. and Essam, D. (2015a) ‘A recovery model for a supply chain 
system with multiple suppliers subject to supply disruption', Journal of Engineering Science and Technology, 10, pp. 89-101.

Hishamuddin, H., Sarker, R. and Essam, D. (2015b) 'A simulation model of a three echelon supply chain system with multiple suppliers subject to supply and transportation disruptions', IFAC Proceedings Volumes (IFAC-PapersOnline). Elsevier Ltd., 48(3), pp. 2036-2040. doi: 10.1016/j.ifacol.2015.06.388.

Hou, J., Zeng, A. Z. and Zhao, L. (2010) 'Coordination with a backup supplier through buyback contract under supply disruption', Transportation Research Part E: Logistics and Transportation Review. Elsevier Ltd, 46(6), pp. 881-895. doi: 10.1016/j.tre.2010.03.004.

Huang, H., Zeng, N. and Xu, H. (2018) 'Procurement mechanism for dual sourcing and emergency production under capacity constraint', Computers and Industrial Engineering. Elsevier, 119, pp. 204-218. doi: 10.1016/j.cie.2018.03.019.

Huber, C., Finelli, L. and Stevens, W. (2018) 'The economic and social burden of the 2014 Ebola outbreak in West Africa', The Journal of Infectious Diseases, 218(Supplement_5), pp. 698-704.

Hudecheck, M. et al. (2020) 'How companies can respond to the coronavirus', MIT Sloan Management Review.

Iswara, A. (2020) '35 manufacturers ramp up capacity to produce COVID-19 protective gear', The Jakarta Post, 7 April.

Ivanov, D. et al. (2016) 'Disruption-driven supply chain (re)-planning and performance impact assessment with consideration of pro-active and recovery policies', Transportation Research Part E: Logistics and Transportation Review. Elsevier Ltd, 90, pp. 7-24. doi: 10.1016/j.tre.2015.12.007.

Ivanov, D. et al. (2017) 'Literature review on disruption recovery in the supply chain', International Journal of Production Research. Taylor \& Francis, 55(20), pp. 6158-6174. doi: 10.1080/00207543.2017.1330572.

Ivanov, D. (2019) 'Disruption tails and revival policies: A simulation analysis of supply chain design and production-ordering systems in the recovery and post-disruption periods', Computers and Industrial Engineering. Elsevier, 127, pp. 558-570. doi: 10.1016/j.cie.2018.10.043. 
Ivanov, D. (2020a) 'Predicting the impacts of epidemic outbreaks on global supply chains: a simulation-based analysis on the coronavirus outbreak (COVID-19/SARS-CoV-2) case', Transportation Research Part E: Logistics and Transportation Review. Elsevier, 136, p. 101922. doi: 10.1016/j.tre.2020.101922.

Ivanov, D. (2020b) 'Viable supply chain model: integrating agility, resilience and sustainability perspec- tives. Lessons from and thinking beyond the COVID-19 pandemic', Annals of Operations Research.

Ivanov, D. and Dolgui, A. (2020a) 'A digital supply chain twin for managing the disruption risks and resilience in the era of Industry 4.0', Production Planning \& Control.

Ivanov, D. and Dolgui, A. (2020b) 'Viability of intertwined supply networks: extending the supply chain resilience angles towards survivability. a position paper motivated by COVID19 outbreak', International Journal of Production Research. Taylor \& Francis, pp. 1-12. doi: 10.1080/00207543.2020.1750727.

Ivanov, D., Dolgui, A. and Sokolov, B. (2019) 'The impact of digital technology and Industry 4.0 on the ripple effect and supply chain risk analytics', International Journal of Production Research. Taylor \& Francis, 57(3), pp. 829-846. doi: 10.1080/00207543.2018.1488086.

Ivanov, D., Pavlov, A. and Sokolov, B. (2014) 'Optimal distribution (re)planning in a centralized multi-stage supply network under conditions of the ripple effect and structure dynamics', European Journal of Operational Research, 237(2), pp. 758-770. doi: 10.1016/j.ejor.2014.02.023.

Ivanov, D., Sokolov, B. and Pavlov, A. (2013) 'Dual problem formulation and its application to optimal redesign of an integrated production-distribution network with structure dynamics and ripple effect considerations', International Journal of Production Research, 51(18), pp. 5386-5403. doi: 10.1080/00207543.2013.774503.

Johanis, D. (2007) 'How Toronto Pearson International Airport applied lessons from SARS to develop a pandemic response plan', Journal of Business Continuity \& Emergency Planning, 1(4), pp. 356-368.

Koonin, L. M. (2020) 'Novel coronavirus disease (COVID-19) outbreak: now is the time to refresh pandemic plans', Journal of Business Continuity \& Emergency Planning, 13(4), pp. $1-15$. 
Koyuncu, M. and Erol, R. (2010) 'Optimal resource allocation model to mitigate the impact of pandemic influenza: a case study for Turkey', Journal of Medical Systems, 34(1), pp. 6170. doi: 10.1007/s 10916-008-9216-y.

Laing, T. (2020) 'The economic impact of the Coronavirus 2019 (Covid-2019): implications for the mining industry', The Extractive Industries and Society. Elsevier Ltd. doi: 10.1016/j.exis.2020.04.003.

Lavastre, O., Gunasekaran, A. and Spalanzani, A. (2012) 'Supply chain risk management in French companies', Decision Support Systems, 52(4), pp. 828-838.

Lee, H. L. et al. (2020) 'The effects of COVID-19 on global supply chains: responsiveness, resilience, and restoration (3Rs): Special issue call for papers', Journal of Operations Management.

Linton, T. and Vakil, B. (2020) 'Coronavirus is proving we need more resilient supply chains', Harvard Business Review.

Lücker, F., Seifert, R. W. and Biçer, I. (2019) 'Roles of inventory and reserve capacity in mitigating supply chain disruption risk', International Journal of Production Research. Taylor \& Francis, 57(4), pp. 1238-1249. doi: 10.1080/00207543.2018.1504173.

Mogaji, E. (2020) 'Financial vulnerability during a pandemic: insights for coronavirus disease (COVID-19)', SSRN Electronic Journal, 2020(5), pp. 57-63. doi: 10.2139/ssrn.3564702.

Norrman, A. and Jansson, U. (2004) 'Ericsson's proactive supply chain risk management approach after a serious sub-supplier accident', International Journal of Physical Distribution \& Logistics Management, 34(5), pp. 434-456. doi: doi:10.1108/09600030410545463.

Pal, B., Sana, S. S. and Chaudhuri, K. (2014) 'A multi-echelon production-inventory system with supply disruption', Journal of Manufacturing Systems. The Society of Manufacturing Engineers, 33(2), pp. 262-276.

Paul, S. K., Sarker, R., et al. (2019) A mathematical modelling approach for managing sudden disturbances in a three-tier manufacturing supply chain, Annals of Operations Research. Springer US. doi: 10.1007/s10479-019-03251-w.

Paul, S. K., Asian, S., et al. (2019) 'Managing sudden transportation disruptions in supply 
chains under delivery delay and quantity loss', Annals of Operations Research, 273(1-2), pp. 783-814. doi: 10.1007/s10479-017-2684-z.

Paul, Sanjoy Kumar, Sarker, R. and Essam, D. (2014) 'Managing real-time demand fluctuation under a supplier-retailer coordinated system', International Journal of Production Economics, 158, pp. 231-243.

Paul, S.K., Sarker, R. and Essam, D. (2014) 'Real time disruption management for a twostage batch production-inventory system with reliability considerations', European Journal of Operational Research, 237(1), pp. 113-128. doi: 10.1016/j.ejor.2014.02.005.

Paul, S. K., Sarker, R. and Essam, D. (2015a) 'A disruption recovery plan in a three-stage production-inventory system', Computers and Operations Research, 57, pp. 60-72. doi: 10.1016/j.cor.2014.12.003.

Paul, S. K., Sarker, R. and Essam, D. (2015b) 'Managing disruption in an imperfect production-inventory system', Computers \& Industrial Engineering, 84, pp. 101-112. doi: 10.1016/j.cie.2014.09.013.

Paul, S. K., Sarker, R. and Essam, D. (2016) 'A reactive mitigation approach for managing supply disruption in a three-tier supply chain', Journal of Intelligent Manufacturing, pp. 117. doi: 10.1007/s 10845-016-1200-7.

Paul, S. K., Sarker, R. and Essam, D. (2017) 'A Quantitative Model for Disruption Mitigation in a Supply Chain', European Journal of Operational Research, 257(3), pp. 881-895.

Paul, S. K. and Shams, R. (2018) 'A quantitative and simulation model for managing sudden supply delay with fuzzy demand and safety stock', International Journal of Production Research, 56(13), pp. 4377-4395.

Pavlov, A., Ivanov, D., Werner, F., et al. (2019) 'Integrated detection of disruption scenarios, the ripple effect dispersal and recovery paths in supply chains', Annals of Operations Research. Springer US, pp. 1-23. doi: 10.1007/s10479-019-03454-1.

Pavlov, A., Ivanov, D., Pavlov, D., et al. (2019) 'Optimization of network redundancy and contingency planning in sustainable and resilient supply chain resource management under conditions of structural dynamics', Annals of Operations Research. Springer US, pp. 1-30. doi: 10.1007/s10479-019-03182-6.

Peck, H. (2005) 'Drivers of supply chain vulnerability: an integrated framework', 
International Journal of Physical Distribution \& Logistics Management, 35(4), pp. 210-232.

Safaeian, M. et al. (2019) 'A multi-objective supplier selection and order allocation through incremental discount in a fuzzy environment', Journal of Intelligent and Fuzzy Systems, 37(1), pp. 1435-1455. doi: 10.3233/JIFS-182843.

Sawik, T. (2019) 'Disruption mitigation and recovery in supply chains using portfolio approach', Omega. Elsevier Ltd, 84, pp. 232-248. doi: 10.1016/j.omega.2018.05.006.

Shao, X.-F. and Dong, M. (2012) 'Supply Disruption and Reactive Strategies in an Assemble-to-Order Supply Chain With Time-Sensitive Demand', IEEE Transactions on Engineering Management, 59(2), pp. 201-212.

Shao, X. F. (2012) 'Demand-side reactive strategies for supply disruptions in a multipleproduct system', International Journal of Production Economics. Elsevier, 136(1), pp. 241252. doi: 10.1016/j.ijpe.2011.11.024.

Silbermayr, L. and Minner, S. (2014) 'A multiple sourcing inventory model under disruption risk', International Journal of Production Economics. Elsevier, 149, pp. 37-46. doi: 10.1016/j.ijpe.2013.03.025.

Tang, C. S. (2006) 'Perspectives in supply chain risk management', International Journal of Production Economics, 103(2), pp. 451-488. doi: 10.1016/j.ijpe.2005.12.006.

Thun, J.-H. and Hoenig, D. (2011) 'An empirical analysis of supply chain risk management in the German automotive industry', International Journal of Production Economics, 131(1), pp. 242-249. doi: 10.1016/j.ijpe.2009.10.010.

Vergragt, P., Akenji, L. and Dewick, P. (2014) 'Sustainable production, consumption, and livelihoods: Global and regional research perspectives', Journal of Cleaner Production, 63, pp. 1-12.

WHO (2020) WHO Director-General's opening remarks at the media briefing on COVID-19. Available at: https://www.who.int/dg/speeches/detail/who-director-general-s-openingremarks-at-the-media-briefing-on-covid-19---11-march-2020 (Accessed: 25 April 2020).

Wilson, M. C. (2007) 'The impact of transportation disruptions on supply chain performance', Transportation Research Part E: Logistics and Transportation Review, 43(4), pp. 295-320. 
Zsidisin, G. A. (2003) 'A grounded definition of supply risk', Journal of Purchasing and Supply Management, 9(5), pp. 217-224. 